\section{( OPEN ACCESS}

\title{
Rest and other types of tremor in adult-onset primary dystonia
}

\author{
Roberto Erro, ${ }^{1}$ Ignacio Rubio-Agusti, ${ }^{1,2}$ Tabish A Saifee, ${ }^{1}$ Carla Cordivari, ${ }^{3}$ \\ Christos Ganos, ${ }^{1,4}$ Amit Batla, ${ }^{1}$ Kailash P Bhatia ${ }^{1}$
}

\begin{abstract}
${ }^{1}$ Sobell Department of Motor Neuroscience and Movement Disorders, University College London (UCL) Institute of Neurology, London, UK ${ }^{2}$ Movement Disorders Unit, Neurology Department, Hospital Universitari La Fe, Valencia, Spain

${ }^{3}$ Department of Clinical Neurophysiology, National Hospital for Neurology and Neurosurgery, London, UK ${ }^{4}$ Department of Neurology, University Medical Center Hamburg-Eppendorf (UKE), Hamburg, Germany
\end{abstract}

\section{Correspondence to} Dr Kailash P Bhatia, Sobell Department of Motor Neuroscience and Movement Disorders, UCL Institute of Neurology, 7 Queen Square, London WC1N 3BG, UK: k.bhatia@ucl.ac.uk

RE and IR-A contributed equally to this study.

Received 16 May 2013 Revised 6 September 2013 Accepted 12 October 2013 Published Online First 18 November 2013

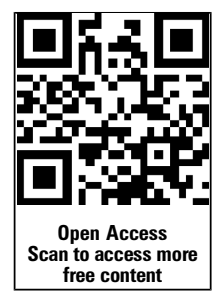

\section{SLinked}

- http://dx.doi.org/10.1136/ jnnp-2013-306538

To cite: Erro R, RubioAgusti I, Saifee TA, et al. J Neurol Neurosurg Psychiatry 2014;85:965-968.

\section{ABSTRACT}

Introduction Knowledge regarding tremor prevalence and phenomenology in patients with adult-onset primary dystonia is limited. Dystonic tremor is presumably underreported, and we aimed to assess the prevalence and the clinical correlates of tremor in patients with adultonset primary dystonia.

Methods We enrolled 473 consecutive patients with different types of adult-onset primary dystonia. They were assessed for presence of head tremor and arm tremor (rest, postural and kinetic).

Results A total of 262 patients (55.4\%) were tremulous: 196 patients presented head tremor, 140 patients presented arm tremor and 98 of them had a combination of head and arm tremor. Of the 140 patients with arm tremor, all presented postural tremor, 103 patients (73.6\%) presented also a kinetic component, whereas 57 patients (40.7\%) had rest tremor. Rest tremor was unilateral/asymmetric in up to $92.9 \%$ of them. Patients with segmental and multifocal dystonia were more likely tremulous than patients with focal dystonia. Dystonic symptoms involving the neck were more frequently observed in patients with head tremor, whereas dystonic symptoms involving the arms were more frequently observed in patients with arm tremor.

Discussion Here we show that tremor is a common feature of patients with adult-onset primary dystonia. It may involve different body segments, with the head being the most commonly affected site. Arm tremor is also frequent (postural>kinetic $>$ rest), occurring in up to one-third of cases. There is a suggestion of a stronger tendency for spread of dystonic features in patients with associated tremor. Dystonic tremor is under-reported and this underscores the importance of careful clinical examination when assessing tremulous patients without overt dystonia.

\section{INTRODUCTION}

Rest tremor is defined by tremor that occurs in a body part which is completely supported against gravity. It is mostly found in Parkinson's disease (PD) but can be less frequently seen in other conditions, such as essential tremor (ET) and dystonia. ${ }^{1}$ Although there is a consensus stating that tremor and dystonia can be associated, ${ }^{1}$ little is known regarding tremor prevalence and phenomenology in patients with adult onset primary dystonia (AOPD) and only one study has focused on its characteristics in a large cohort of patients with AOPD so far. ${ }^{2}$ The authors found that tremor is a relatively common feature, being postural or postural/ kinetic in nature and occurring in about $17 \%$ of patients with AOPD. Interestingly, none of their patients had rest tremor, thus suggesting that it is an almost non-existent phenomenon in AOPD. However, this is not our experience. ${ }^{3-5}$ We have indeed identified rest tremor in a number of our patients with cervical and other forms of adult onset dystonia, ${ }^{3-5}$ but literature on dystonic tremor is still poor.

The present study was therefore designed to assess prevalence and clinical correlates of rest and other types of tremor in a large cohort of patients with AOPD.

\section{PATIENTS AND METHODS}

We prospectively assessed consecutive patients with a diagnosis of AOPD attending the botulinum toxin clinic at the National Hospital for Neurology and Neurosurgery, London, UK, between April 2010 and December 2012. Part of this cohort (approximately 55\%) has been already been described. ${ }^{6}$ This study is selectively based on the clinical information and investigations carried out as part of the routine clinical care of the patients. It has been therefore structured as a clinical audit and, as such, our institution does not require ethics committee approval. All patients had a clinical diagnosis of primary dystonia according to current criteria. ${ }^{7}$ Patients with secondary dystonia were not included. For the current audit, we included patients, who have been assessed by one of the authors at least 3 months after their last set of injections. Patients have been classified as having focal dystonia (cervical dystonia, cranial dystonia, or writer's cramp); segmental dystonia (cranio-cervical dystonia or neck/arm dystonia), and multifocal dystonia (arm dystonia plus cranial involvement). ${ }^{7}$ None of them had lower-limb involvement. The assessment of tremor included observation of head tremor (seated upright, with the head in neutral position and while turning the head to either side), and arm tremor (rest tremor-arms relaxed in the lap; postural tremor-arms outstretched in pronation and supination; flexed postural tremor-shoulders abducted and elbows flexed, placing both hands, palms down, in front of the chest; kinetic tremorperforming the finger-to-nose manoeuver, writing a standardised sentence and drawing a spiral). Each condition lasted approximately $15 \mathrm{~s}$. If tremor was present for at least $50 \%$ of each condition, patients were classified as tremulous, accordingly. Following current recommendations, ${ }^{1}$ tremor was defined as a rhythmic/semirhythmic involuntary oscillatory 
movement of a body part. Irregular amplitudes and superimposed jerks, recognised as characteristic of dystonic tremor, ${ }^{1}$ were accepted, but patients with isolated head jerks, were not classified as tremulous.

Statistical analyses were performed with the STATA software, V.11.0 (StataCorp LP, USA), using $t$ test, $\chi^{2}$ test, or analysis of variance as appropriate, and deeming $\mathrm{p}$ values of less than 0.05 statistically significant.

\section{RESULTS}

A total of 473 patients with AOPD were included in the current study. Two hundred and sixty-seven patients had focal dystonia (207 patients with cervical dystonia, 32 patients with cranial dystonia and 28 patients with writer's cramp), 135 patients had segmental dystonia (54 patients had cranio-cervico dystonia and 81 patients had neck and arm involvement) and 71 patient had multifocal dystonia (cranial dystonia plus arm involvement). Demographic and clinical characteristics are detailed in table 1.

A total of 262 patients $(55.4 \%)$ were classified as tremulous: 140 patients $(29.6 \%$ of the entire cohort) presented arm tremor, and 196 patients (41.4\% of the entire cohort) presented head tremor. Among tremulous patients, approximately one-third (98 patients) had a combination of head and arm tremor. Of the 140 patients with arm tremor, all presented postural tremor, 103 patients $(73.6 \%)$ presented also a kinetic component, whereas 57 patients $(40.7 \%)$ had rest tremor. Among the latter, rest tremor was unilateral in 40 patients $(70.2 \%)$ and bilateral in 17 patients (29.8\%), of whom 13 (76.5\%) showed an asymmetric tremor. All types of tremor were found to be more frequent in patients with multifocal and segmental dystonia than in patients with focal dystonia $(p<0.01$, table 1$)$. Among those with focal dystonia, head and arm tremor were more prevalent in patients with cervical dystonia than in patients with cranial dystonia and with writer's cramp $(48.3 \%$ vs $21.8 \% 12 \%$, respectively, $\chi^{2}$ test, $\left.\mathrm{p}<0.01\right)$. Details on the pattern of dystonic symptoms in relation to localisation of tremor are given in table 2.

Dystonic symptoms involving the neck were more frequent in patients with head tremor, whereas dystonic symptoms involving the arms were more frequent in patients with arm tremor $(\mathrm{p}<0.01$, table 2$)$. The two groups did not differ with regard to age, disease duration and gender distribution.

\section{DISCUSSION}

Tremor is a common feature among patients with AOPD, with arm and head tremor occurring in $30 \%$ and $40 \%$ of cases,
Table 2 Clinical characteristic of our patients according to localisation of tremor

\begin{tabular}{lllll}
\hline & $\begin{array}{l}\text { Patients } \\
\text { with head } \\
\text { tremor }\end{array}$ & $\begin{array}{l}\text { Patients } \\
\text { with arm } \\
\text { tremor }\end{array}$ & $\begin{array}{l}\text { Patients with } \\
\text { head and } \\
\text { arm tremor }\end{array}$ & $\begin{array}{l}\text { p } \\
\text { Value }\end{array}$ \\
\hline $\begin{array}{l}\text { Number } \\
\text { Sex (F:M) }\end{array}$ & 98 & 42 & 98 & - \\
$\begin{array}{l}\text { Age, years, mean } \\
\text { (SD) }\end{array}$ & $76: 22$ & $33: 9$ & $78: 20$ & 0.19 \\
$\begin{array}{l}\text { Age at onset, years, } \\
\text { mean (SD) }\end{array}$ & $43.18(12.8)$ & $46.3(11.8)$ & $45.9(12.1)$ & 0.29 \\
$\begin{array}{l}\text { Disease duration, } \\
\text { years, mean (SD) }\end{array}$ & $17.2(12.9)$ & $16.7(13.2)$ & $15.9(12.5)$ & 0.65 \\
$\begin{array}{l}\text { Distribution of dystonia } \\
\text { Cervical, n (\%) }\end{array}$ & $75(76.5)$ & $65(45.7)$ & $79(80.6)$ & 0.0001 \\
$\begin{array}{l}\text { Cranial, n (\%) } \\
\text { Brachial, n (\%) }\end{array}$ & $\begin{array}{l}23(23.4) \\
24(24.4)\end{array}$ & $\begin{array}{l}11(26.2) \\
26(61.9)\end{array}$ & $22(22.4)$ & 0.13 \\
\hline
\end{tabular}

respectively. Previous studies have reported tremor frequencies ranging from $7 \%$ to $70 \%$ in AOPD. ${ }^{2}{ }^{8-10}$ Such a huge discrepancy between studies might be due to several factors, including small patient samples, with focus on only one type of focal dystonia, and use of medical chart reviews rather than clinical examination to detect tremor. Intriguingly, the largest study which specifically addressed this question in 429 AOPD patients, found postural or postural/kinetic tremor in $16.7 \%$ of the cases, while none had rest tremor. ${ }^{2}$ We do not believe that methodological issues can explain such discrepancy: similarly to Defazio et al, we have, in fact, clinically assessed consecutive AOPD patients, with the specific aim to look for tremor and its characteristics.

Dystonic tremor is a relatively new category in tremor classification. ${ }^{1}$ It is very likely under-recognised and might be mistaken as ET or even PD, depending on the tremor type and distribution. This relies on the lack of markers for the differential diagnosis of tremulous condition, and highlights the difficulty with the clinical diagnosis of dystonia. Among tremulous disorders, ET is undoubtedly overdiagnosed. ${ }^{11}$ In fact, a review of 350 patients supposed to have 'pure ET' has disclosed associated dystonia, including cervical dystonia, writer's cramp, spasmodic dysphonia, and cranial dystonia, in half of them. ${ }^{12}$ The suggestion that dystonic patients are frequently misdiagnosed as ET has been further confirmed by others. ${ }^{13}$ This may also depend on the fact that tremulous dystonic patients may have such

Table 1 Clinical characteristic of our patients

\begin{tabular}{|c|c|c|c|c|}
\hline & Focal dystonia & Segmental dystonia & Multifocal dystonia & p Value \\
\hline Number of patients & $\begin{array}{l}\text { Total } 267 \\
\text { Cervical dystonia } 207 \\
\text { Cranial dystonia } 32 \\
\text { Writer's cramp } 28\end{array}$ & $\begin{array}{l}\text { Total } 145 \\
\text { Cervico-cranial dystonia } 54 \\
\text { Cranial/neck/arm } 91\end{array}$ & Total 61 & - \\
\hline Age at onset (years, mean \pm SD) & $42.3 \pm 11.9$ & $40.7 \pm 14.3$ & $41.6 \pm 12.5$ & 0.45 \\
\hline Disease duration (years, mean $\pm S D$ ) & $14.7 \pm 8.7$ & $21.4 \pm 10.1$ & $23.5 \pm 9.3$ & 0.09 \\
\hline Sex (female/male) & $203 / 64$ & $109 / 36$ & $42 / 19$ & 0.63 \\
\hline Head tremor $(n)$ & 72 & 80 & 44 & 0.0001 * \\
\hline Arm tremor $(n)$ & $\begin{array}{l}\text { Total } 38 \\
\text { Postural } 38 \text { (asymmetric in 18) } \\
\text { Kinetic } 24 \text { (asymmetric in 20) } \\
\text { Rest } 11 \text { (asymmetric in 10) }\end{array}$ & $\begin{array}{l}\text { Total } 74 \\
\text { Postural } 74 \text { (asymmetric in 45) } \\
\text { Kinetic } 58 \text { (asymmetric in 48) } \\
\text { Rest } 28 \text { (asymmtetric in 26) }\end{array}$ & $\begin{array}{l}\text { Total } 28 \\
\text { Postural } 28 \text { (asymmetric in 14) } \\
\text { Kinetic } 21 \text { (asymmetric in 17) } \\
\text { Rest } 18 \text { (asymmetric in 17) }\end{array}$ & $\begin{array}{l}0.0001 \text { * } \\
0.0001 \text { * } \\
0.0001 \text { * } \\
0.0001^{*}\end{array}$ \\
\hline
\end{tabular}

*Segmental and multifocal versus focal. 
confounding clues as autosomal dominant familial history and alcohol responsiveness. ${ }^{6}$

With regard to head tremor, Agnew et $a l^{14}$ have recently shown that in ET cases it may represent a postural tremor that dissipates when patients lie down, whereas in patients with cervical dystonia (CD), head tremor more often persists. Such a phenomenon was not tested during our audit, but we think it may be unwise to consider ET in subjects with isolated head tremor, or in whom head tremor is more severe than their arm tremor. ${ }^{15}$ In fact, Louis et al studied the prevalence of 'isolated head tremor' among 583 ET patients, and did not identify any case of head tremor in complete absence of arm tremor, and only $2.7 \%$ of cases with head tremor and only mild arm tremor. ${ }^{16}$ This confirms previous findings by Bain et $a l^{17}$ among 20 families with three-generational pure ET. Patients with PD may seldom present with head tremor, but it is usually not a feature of PD and has been very rarely described. ${ }^{18}$ Moreover, we have demonstrated that head tremor is much more common in dystonic patients than in PD cases. ${ }^{5}$ All these evidences suggest that head tremor is rather suggestive of concomitant dystonia.

With a view to rest tremor, it is highly suggestive of PD, but here we show that it may occur in up to $40 \%$ of tremulous dystonic patients (meaning approximately $12 \%$ of all patients with AOPD), being unilateral in more than two-thirds of them. In the majority of them (92.9\%), rest tremor was asymmetric, if not unilateral. This result is in line with our previous observations that a number of patients with unilateral rest tremor and preserved dopaminergic nigrostriatal pathway (ie, patients supposed to be affected with PD, but having scans without evidence of dopaminergic deficit-SWEDDs), have dystonic tremor as per the consensus classification ${ }^{4}$ and also by electrophysiology. ${ }^{5}$ Such tremor characteristics as frequency and amplitude seem to be not useful to differentiate between these two conditions (PD and dystonic patients may indeed both show a tremor at about 5-6 Hz). ${ }^{5}$ However, it is remarkable that none of our patients had re-emergent tremor (ie, a postural tremor that emerges after a variable latency of several seconds) while this phenomenon is commonly seen in patients with PD. On the other hand, rest tremor can be seldom seen in ET, and the existence of an ET-subgroup with associated rest tremor has been suggested. ${ }^{1} 10$ However, a recent study has shown that ET patients with associated rest tremor display a blink reflex recover cycle similar to that detected in dystonic tremor, thus indicating that they may have a dystonic tremor rather than a subtype of ET. ${ }^{19}$ Once again, this evidence suggests that tremulous dystonic patients are likely misdiagnosed. This may depend on the fact that dystonic features can be mild and involve body parts not affected with tremor, or even that have not yet appeared. ${ }^{15}$

When we compared patients with head versus arm tremor regardless of dystonia classification, we found similar age, disease duration and sex distribution. We also found that dystonic symptoms more frequently involved body regions affected by tremor, suggesting that these two phenomena often coexist. On the other hand, tremor involving body regions which were not affected by dystonic symptoms (ie, 'tremor associated with dystonia' as per consensus classification ${ }^{1}$ ), was less frequently observed.

In our cohort, we have found patients with segmental or multifocal dystonia to be more likely tremulous than patients with focal dystonia. This suggests a stronger tendency for spread of dystonic features in patients with tremor, also in line with previous reports. ${ }^{2} 6$ However, whether the presence of tremor in a body segment may anticipate the spread of dystonia in that segment needs to be elucidated in further prospective studies. Moreover, patients with segmental and multifocal dystonia had a longer disease duration (21 and 23 years, respectively), than patients with focal dystonia (15 years), even though this was not significant $(p=0.09)$. On the other hand, tremulous patients had a longer disease duration regardless of the dystonia classification (data not shown), in line with existing literature. ${ }^{7}$ This may suggest that tremor can be a relatively late symptom in patients with AOPD. The long disease duration across the subgroups may also partly account for the discrepancy with previous observations which have included patients with shorter disease duration. ${ }^{7}$ However, we miss data regarding tremor versus dystonia onset. We therefore cannot totally exclude that some patients have developed dystonic symptoms after tremor onset. Moreover, the possibility exists that, at least in some patients, dystonia and tremor are unrelated phenomena, leading to an overestimation bias. However, we believe this is unlikely, given the large sample size.

Neurologists and other practitioners should be aware of the possibility of dystonic tremor. It is frequent in patients with AOPD, with the head being the most common affected site. Arm tremor occurs in one-third of AOPD patients, with or without associated head tremor: arm tremor usually features a postural component, but can be also characterised by kinetic and rest components in some cases. Rest tremor presents more frequently unilaterally (and it is usually asymmetric in patients with bilateral rest tremor), thus it would be wise to consider dystonia even in the differential diagnosis of PD. Our clinical experience would suggest that such features as position/task specificity, jerkiness, presence of tremor-flurries and thumb hyperextension are fairly typical for dystonic tremor. ${ }^{5} 15$

We acknowledge some limitations. First, we did not perform electrophysiological recordings, and it may be argued that rest tremor seen in our patients (which was assessed with the arms in semisupination/pronation on their lap) is actually postural tremor that continues when the limb is supported. However, this is a matter of further laboratory research and does not correspond to what is seen in clinical practice. It may be also argued that some patients, particularly those with head tremor, may adopt abnormal head postures in order to compensate or suppress their tremor. This might mislead to the diagnosis of dystonia, but we do not believe this is the case here. Our patients were in fact diagnosed according to established clinical criteria, ${ }^{7}$ and all our CD cases showed restricted head motion due to paradoxical muscle overactivity, which is suggestive of underlying dystonia. As a tertiary centre for movement disorders, we cannot exclude a referral bias. However, we do not believe that this may account for the whole discrepancy between ours and others studies. We would like to remark the importance of careful clinical examination when assessing tremulous patients, looking for dystonic symptoms also in non-tremulous body segments.

Contributors RE: conception, execution, writing the first draft. IR-A: conception, execution, revising the paper. TAS: execution, revising the paper. CC: execution, revising the paper. CG: execution, revising the paper. $\mathrm{AB}$ : execution, revising the paper. KPB: conception, supervision, revising the paper.

Competing interests KPB receives royalties from the publication of Oxford Specialist Handbook Of Parkinson's Disease and Other Movement Disorders (Oxford University Press, 2008) and received funding for travel from GlaxoSmithKline (GSK), Orion Corporation, Ipsen and Merz Pharmaceuticals. All other authors have no conflicts of interest.

Provenance and peer review Not commissioned; externally peer reviewed.

Open Access This is an Open Access article distributed in accordance with the Creative Commons Attribution Non Commercial (CC BY-NC 3.0) license, which permits others to distribute, remix, adapt, build upon this work non-commercially, and license their derivative works on different terms, provided the original work is properly cited and the use is non-commercial. See: http://creativecommons.org/ licenses/by-nc/3.0/ 


\section{REFERENCES}

1 Deuschl G, Bain P, Brin M. Consensus statement of the Movement Disorder Society on Tremor. Ad Hoc Scientific Committee. Mov Disord 1998;13:2-23.

2 Defazio G, Gigante AF, Abbruzzese G, et al. Tremor in primary adult-onset dystonia: prevalence and associated clinical features. J Neurol Neurosurg Psychiatry 2013;84:404-8.

3 Erro R, Quinn NP, Schneider SA, et al. Does rest tremor exclude the diagnosis of adult-onset primary dystonia? J Neurol Neurosurg Psychiatry 2013;84:708.

4 Schneider SA, Edwards MJ, Mir P, et al. Patients with adult-onset dystonic tremor resembling parkinsonian tremor have scans without evidence of dopaminergic deficit (SWEDDs). Mov Disord 2007;22:2210-15.

5 Schwingenschuh P, Ruge D, Edwards MJ, et al. Distinguishing SWEDDs patients with asymmetric resting tremor from Parkinson's disease: a clinical and electrophysiological study. Mov Disord 2010;25:560-9.

6 Rubio-Agusti I, Pareés I, Kojovic M, et al. Tremulous cervical dystonia is likely to be familial: Clinical characteristics of a large cohort. Parkinsonism Relat Disord 2013;19:634-8.

7 Albanese A, Bhatia KP, Bressman SB, et al. Phenomenology and Classification of Dystonia: A Consensus Update. Mov Disord 2013;28:863-73.

8 Deuschl G, Heinen F, Guschlbauer B, et al. Hand tremor in patients with spasmodic torticollis. Mov Disord 1997;12:547-52.

9 Jedynak CP, Bonnet AM, Agid Y. Tremor and idiopathic dystonia. Mov Disord 1991;6:230-6.
10 Jankovic J, Leder S, Warner D, et al. Cervical dystonia: clinical findings and associated movement disorders. Neurology 1991;41:1088-91.

11 Schrag A, Muenchau A, Bhatia KP, et al. Overdiagnosis of essential tremor. Lancet 1999;353:1498-9.

12 Lou JS, Jankovic J. Essential tremor: clinical correlates in 350 patients. Neurology 1991:41:234-8.

13 Louis ED, Hernandez N, Alcalay RN, et al. Prevalence and features of unreported dystonia in a family study of "pure" essential tremor. Parkinsonism Relat Disord 2013;19:359-62.

14 Agnew A, Frucht SJ, Louis ED. Supine head tremor: a clinical comparison of essential tremor and spasmodic torticollis patients. J Neurol Neurosurg Psychiatry 2012;83:179-81.

15 Quinn NP, Schneider SA, Schwingenschuh P, et al. Tremor-some controversial aspects. Mov Disord 2011;26:18-23.

16 Louis ED, Dogu 0. Isolated head tremor: part of the clinical spectrum of essential tremor? Data from population-based and clinic- based case samples. Mov Disord 2009;24:2281-5

17 Bain PG, Findley LJ, Thompson PD, et al. A study of hereditary essential tremor. Brain 1994:117:805-24.

18 Roze E, Coêlho-Braga MC, Gayraud D, et al. Head tremor in Parkinson's disease. Mov Disord 2006:21:1245-8.

19 Nisticò R, Pirritano D, Novellino F, et al. Blink reflex recovery cycle in patients with essential tremor associated with resting tremor. Neurology 2012;79:1490-5. 